\title{
Guideline:
}

\section{Analysis of an improved workflow of endoscope reprocessing for bedside endoscopic diagnosis and treatment on COVID-19 patients ${ }^{\#}$}

\author{
Qing $\mathrm{GU}^{\dagger 1}$, Hua-fen WANG ${ }^{\dagger 11}$, Ying FANG ${ }^{1}$, Ye $\mathrm{LU}^{3}$, Zhe SHEN ${ }^{2}$, \\ Yan $\mathrm{WANG}^{1}$, Xin WU ${ }^{1}$, Li CEN ${ }^{2}$, Yi-shu CHEN ${ }^{2}$ \\ ${ }^{I}$ Center of Endoscopy, the First Affiliated Hospital, School of Medicine, Zhejiang University, Hangzhou 310003, China \\ ${ }^{2}$ Department of Gastroenterology, the First Affiliated Hospital, School of Medicine, Zhejiang University, Hangzhou 310003, China \\ ${ }^{3}$ Zhejiang Provincial Center for Disease Control and Prevention, Hangzhou 310003, China \\ †E-mail: 1191016@zju.edu.cn; 2185015@zju.edu.cn
}

Received Mar. 10, 2020; Revision accepted Mar. 22, 2020; Crosschecked Mar. 31, 2020; Published online May 9, 2020

\begin{abstract}
Severe cases infected with the coronavirus disease 2019 (COVID-19), named by the World Health Organization (WHO) on Feb. 11, 2020, tend to present a hypercatabolic state because of severe systemic consumption, and are susceptible to stress ulcers and even life-threatening gastrointestinal bleeding. Endoscopic diagnosis and treatment constitute an irreplaceable part in the handling of severe COVID-19 cases. Endoscopes, as reusable precision instruments with complicated structures, require more techniques than other medical devices in cleaning, disinfection, sterilization, and other reprocessing procedures. From 2016 to 2019, health care-acquired infection caused by improper endoscope reprocessing has always been among the top 5 on the list of top 10 health technology hazards issued by the Emergency Care Research Institute. Considering the highly infective nature of COVID-19 and the potential aerosol contamination therefrom, it is of pivotal significance to ensure that endoscopes are strictly reprocessed between uses. In accordance with the national standard "Regulation for Cleaning and Disinfection Technique of Flexible Endoscope (WS507-2016)," we improved the workflow of endoscope reprocessing including the selection of chemicals in an effort to ensure quality control throughout the clinical management towards COVID-19 patients. Based on the experience we attained from the 12 severe COVID-19 cases in our hospital who underwent endoscopy 23 times in total, the article provides an improved version of endoscopic reprocessing guidelines for bedside endoscopic diagnosis and treatment on COVID-19 patients for reference.
\end{abstract}

Key words: Novel coronavirus; Coronavirus disease 2019 (COVID-19); 2019 novel coronavirus (2019-nCoV); Disinfection; Gastrointestinal endoscopy; Infection prevention and control

https://doi.org/10.1631/jzus.B2000109

\section{Introduction}

In Dec. 2019, a new type of pneumonia, named coronavirus disease 2019 (COVID-19) later, was first reported in Wuhan, Hubei Province, China. As the

\footnotetext{
¿ Corresponding author

\# Electronic supplementary materials: The online version of this article (https://doi.org/10.1631/jzus.B2000109) contains supplementary materials, which are available to authorized users

(D) ORCID: Qing GU, https://orcid.org/0000-0003-2292-2893

(C) Zhejiang University and Springer-Verlag GmbH Germany, part of Springer Nature 2020
}

CLC number: R473.5

outbreak evolved, the number of infected cases surged ahead nationwide and overseas (Wu et al., 2020). As of Apr. 3, 2020, a total of 82802 patients have been diagnosed in China, with 3331 deaths and a fatality rate of $4.0 \%$. Real-time data indicate that $26.8 \%$ of the currently existing confirmed cases are in severe condition, usually with dyspnea and/or hypoxemia one or two weeks after onset (Huang et al., 2020). Severe COVID-19 cases, mostly elderly people with underlying diseases, suffered from difficulty in eating with a high risk of regurgitation and aspiration. 
The hypercatabolic state caused by the disease could lead to declined physical functioning and weakened immunity. The stress condition as well as drug-derived factors could induce stress ulcers and even lifethreatening gastrointestinal bleeding, which should be highlighted in the diagnosis and treatment of severe cases. Therefore, in addition to symptomatic treatment, it is essential to strengthen nutritional support and prevent such complications. Endoscopic diagnosis and treatment (including placement of jejunal nutrition tubes and emergency endoscopic treatment of gastrointestinal bleeding) serve as an indispensable part in the management of severe COVID-19 cases. This contributes to a better prognosis and treatment (Gu et al., 2019; Lou et al., 2019). Endoscopes, as reusable precision instruments with complicated structures, require more techniques than other medical devices in cleaning, disinfection, sterilization, and other reprocessing procedures. From 2016 to 2019 inclusive, health care-acquired infection caused by improper endoscope reprocessing was among the top 5 on the list of 2019 top 10 health technology hazards issued by the Emergency Care Research Institute (2018).

Our hospital, the First Affiliated Hospital, School of Medicine, Zhejiang University (Hangzhou, China), designated as one of the health care providers for COVID-19, is responsible for most of the severe cases in Zhejiang Province. By Mar. 9, 2020, we have kept the record of zero deaths and zero infection cases. The gastrointestinal endoscopy center, where health care-acquired infection control is strictly enforced, has paid much attention to infection control and occupational protection in the battle against COVID-19. Based on the endoscope reprocessing guideline proposed by the Chinese Society of Digestive Endoscopology (CSDE, 2020b), we comprehensively summarized the experience we obtained from the 12 COVID19 severe cases who underwent bedside endoscopy 23 times in total (including placement of jejunal nutrition tubes and gastrointestinal bleeding treatment) and provided a report as follows.

\section{Clinical data}

A total of 12 COVID-19 patients rated as severe cases were treated in the intensive care unit (ICU) of our hospital with tracheal intubation and ventilator-assisted ventilation. There were eight males and four females, aged 36-90 years with an average age of 72.8 years. To address such problems as nutrition tube clogging, tube dislocation, and gastrointestinal bleeding, we performed endoscopy on these cases 23 times in total.

\section{Introduction of the Improved workflow for endoscope reprocessing}

\subsection{Specialized training for health workers}

An expert panel was organized by the gastrointestinal endoscopy center to conduct an education and training program for health workers including doctors, nurses, and other endoscope processing personnel. The program was organized in conformity with the guidelines proposed by CSDE (2020a) and the National Health Commission of the People's Republic of China (NHC, 2020a).

\subsection{Special channel for endoscope transport and personal protection for endoscope reprocessing personnel}

Taking account of the layout of the hospital, we set up a transport route specially for endoscopes to ensure infection control. According to the three-level protection requirements (NHFPC, 2017b), technical personnel should get dressed in specialized personal protection equipment (PPE) in the buffer area before entering the endoscope reprocessing area. After the reprocessing work, the personnel should take off their PPE in the buffer area before going back to clean working areas.

\subsection{Preparation for endoscope reprocessing}

1. Bedside pre-processing items: Weak alkaline peroxyacetic acid-sterilizing powder diluent $(0.23 \%$; concentration monitoring required), disposable plastic bags with movable seals filled with $0.23 \%$ peroxyacetic acid, special transport bags for endoscopes, $2500 \mathrm{mg} / \mathrm{L}$ available chlorine disinfectant, yellow plastic bags for medical waste, leakproof transport containers for endoscopes, chlorine-containing disinfection towels, sterile gauze, waterproof markers, etc. (Fig. 1).

2. Endoscope reprocessing items: Weak alkaline peroxyacetic acid-sterilizing powder diluent $(0.23 \%)$, disinfectant concentration test strips, cleaning sink, 
perfusion channels, 50-mL syringes, disposable endoscope cleaning brushes (one for each length), endoscope maintenance device (MU-1, Olympus Inc., Tokyo, Japan), leak testing device (MB-155; Olympus Inc., Tokyo, Japan), automated endoscope reprocessor (AER; Medivators Inc., Minneapolis, USA), yellow plastic bags for medical waste, special transport bags for endoscopes, etc. (Fig. 1).

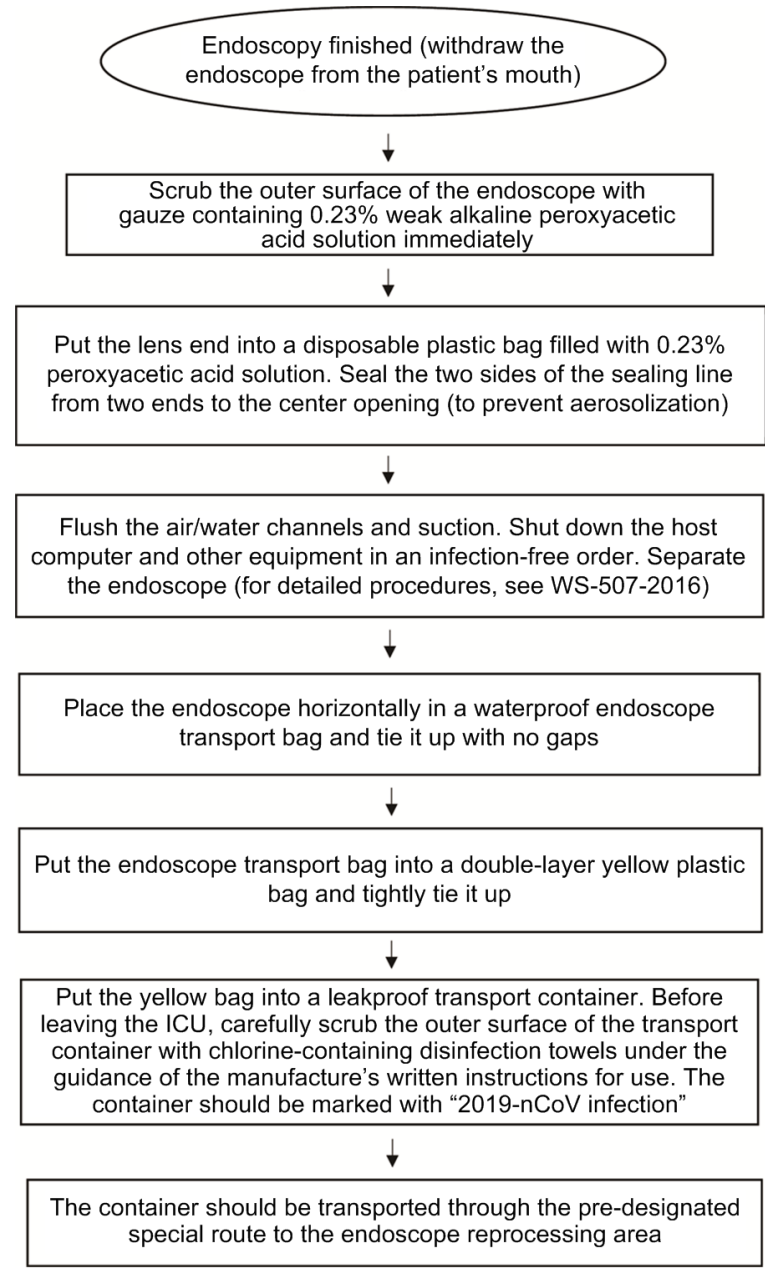

Fig. 1 Workflow of bedside pre-processing of endoscopes contaminated by patients infected with COVID-19

\subsection{Preparation of personal protective equipment}

1. Protective equipment for bedside pre-processing operators: Goggles or protective face-shields, N95 medical protective masks, surgical masks, medical protective caps (or positive-pressure respiratory protective equipment if available), scrubs, impermeable isolation gowns, disposable medical protective clothing, waterproof boot covers, medical shoe covers, double-layer gloves (disposable sterilized surgical gloves on the outer layer), etc.

2. Protective equipment for reprocessing operators: Goggles or protective face-shields, medical protective masks (N95 at least), medical protective caps, scrubs, impermeable waterproof isolation gowns, waterproof aprons, disposable medical protective clothing, waterproof boot covers, medical shoe covers, gloves (long-sleeved thickened rubber gloves), etc.

\subsection{Improved strategies for bedside pre-processing of contaminated endoscopes}

As has been previously reported, the qualified rate of reprocessed endoscopes could be reduced from $99.21 \%$ to $90.75 \%$ if the contaminated instruments are cleaned as late as one hour after surgery compared with immediate cleaning after surgery (Xia et al., 2019). Dryness of instrument lumens caused by delayed cleaning could strongly reduce the effectiveness of reprocessing. This could be avoided by bedside pre-processing so as to maintain moisture in lumens during transport. The sooner the pre-processing is done and the shorter the transport period is, the more effective the reprocessing will be. As the COVID-19 is highly infectious, it has been classified by the state as a Class B infectious disease, which should be managed as a Class A one (NHC, 2020a). As the endoscopes could be tainted by viruses and multi-drug resistant bacteria from the saliva of the COVID-19 cases, immediate bedside pre-processing after use should be highlighted in order to prevent cross infection. For bedside pre-processing of such contaminated endoscopes, it is advisable to upgrade the cleaning agents from conventional enzyme agents, which have no disinfecting or sterilizing effects, to peroxyacetic acid. The pre-disinfecting effect of peroxyacetic acid could reduce the risk of infection in subsequent operations.

We have improved the bedside pre-processing workflow as follows: (1) For bedside endoscopic diagnosis and treatment, ward beds should be placed in an operation-friendly manner. The needs of the facility should determine the size of the operation areas. It is recommended that the ward should provide an operation area with a radius of over $80 \mathrm{~cm}$ for each bed to avoid physical contact with surrounding objects. (2) As soon as the endoscopy is finished by the doctor, the pre-processing operator should immediately scrub 
the outer surface of the endoscope with gauze containing $0.23 \%$ weak alkaline peroxyacetic acid solution to avoid possible hazards of the saliva adherent to the endoscope surface. (3) After scrubbing, put the lens end into a disposable sterilized plastic bag filled with $0.23 \%$ weak alkaline peroxyacetic acid solution (Fig. S1). Then seal the two sides of the sealing line from two ends to the center opening. Flush the air and water channels for $10 \mathrm{~s}$ each, and suction until the tube is free from dirt under visual inspection. These procedures could effectively prevent aerosol contamination during flushing and suctioning, and greatly reduce the risk of occupational exposure. The suctioning liquid should be upgraded to $0.23 \%$ weak alkaline peroxyacetic acid sterilizing powder diluent instead of the conventional enzyme cleaning agents since previous studies have proved that weak alkaline peroxyacetic acid sterilizing powder could not only remove and decompose adherent organics, but also disinfect and sterilize the endoscopes (Gu et al., 2018). (4) Use a disposable or new endoscope knob. If the knob valve is damaged, the risk of air leakage and aerosolization during the flushing and suctioning processes will be substantially elevated. (5) As the qualified rate is closely associated with the transport period, the endoscopes after use should be transported to the reprocessing area as soon as possible. We recommend loading the pre-processed endoscope into a transport bag in an oval shape to avoid contamination or unexpected injuries when operators reopen it in the reprocessing area. Put the endoscope transport bag into a double-layer yellow garbage bag and tightly fasten it. Apply $2500 \mathrm{mg} / \mathrm{L}$ available chlorine disinfectant to the opening and then place it in a leakproof transport container. Before leaving the ICU room, carefully scrub again the outer surface of the transport container with chlorine-containing disinfection towels under the guidance of the manufacturer's written instructions for use. The container should be marked with "COVID-19" and be transported through the special channel to the reprocessing area.

\subsection{Improved strategies for endoscopic cleaning, disinfection, and sterilization}

On the basis of the recommendations by Fang (2011) and CSDE (2020b), our department drew experience from actual clinical practice and provided an improved workflow for reference. The reprocessing procedures follow the order as disinfection first, cleaning next, and then sterilization. Chemicals such as disinfectant and cleaning agent, disposable items such as scrubbing cottons and cleaning brushes, and facilities such as cleaning and rising sinks should all follow the principle of single use. Reprocessing operators dressed in PPE should strictly observe the rules of hand hygiene.

We have improved the reprocessing workflow as follows (Fig. 2): (1) Take out the endoscope from the transport container. All the packaging material should be collected in tightly tied double-layer yellow plastic bags and disposed of as hazardous infectious waste according to relevant standards. (2) Place all components of the endoscope in the sink filled with $0.23 \%$ weak alkaline peroxyacetic acid solution (concentration monitoring required). Make sure that at least the small knob lock of the endoscope operation portion is immersed in the solution. Connect all perfusion channels and inject $0.23 \%$ peroxyacetic acid solution into the channels with a $50-\mathrm{mL}$ syringe until the channels are fully filled (Fig. S2). Let it stand for $5 \mathrm{~min}$. This serves as a pre-high-level disinfecting process. The pre-disinfection prior to routine cleaning process could help reduce the risk of contact and aerosol contamination during the following procedures such as air and water flushing, air gun drying, and channel brushing. Previous studies have proved that aerosols are solid particles or liquid droplets that can be suspended in the air for a long time, and by which many types of pathogens can be transmitted (Bakanidze et al., 2010). Aerosols that carry pathogens may freely float in the air, adhere to equipment surfaces, or be inhaled by health workers, resulting in a higher risk of cross infection. (3) As has been recommended by NHFPC (2017a), after brushing the channels and buttons, inject $0.23 \%$ peroxyacetic acid solution into each channel of the endoscope and keep continuous, dynamic flushing for $5 \mathrm{~min}$ to ensure that the dirt and debris that have been brushed off can be fully washed away. (4) Place the endoscope into an AER for high-level disinfection and then send it to the supply center for ethylene oxide sterilization. (5) According to the opinions from the experts on infection control and endoscopic reprocessing, we recommend that leak testing should be conducted after high-level disinfection to avoid contamination in this special period. 


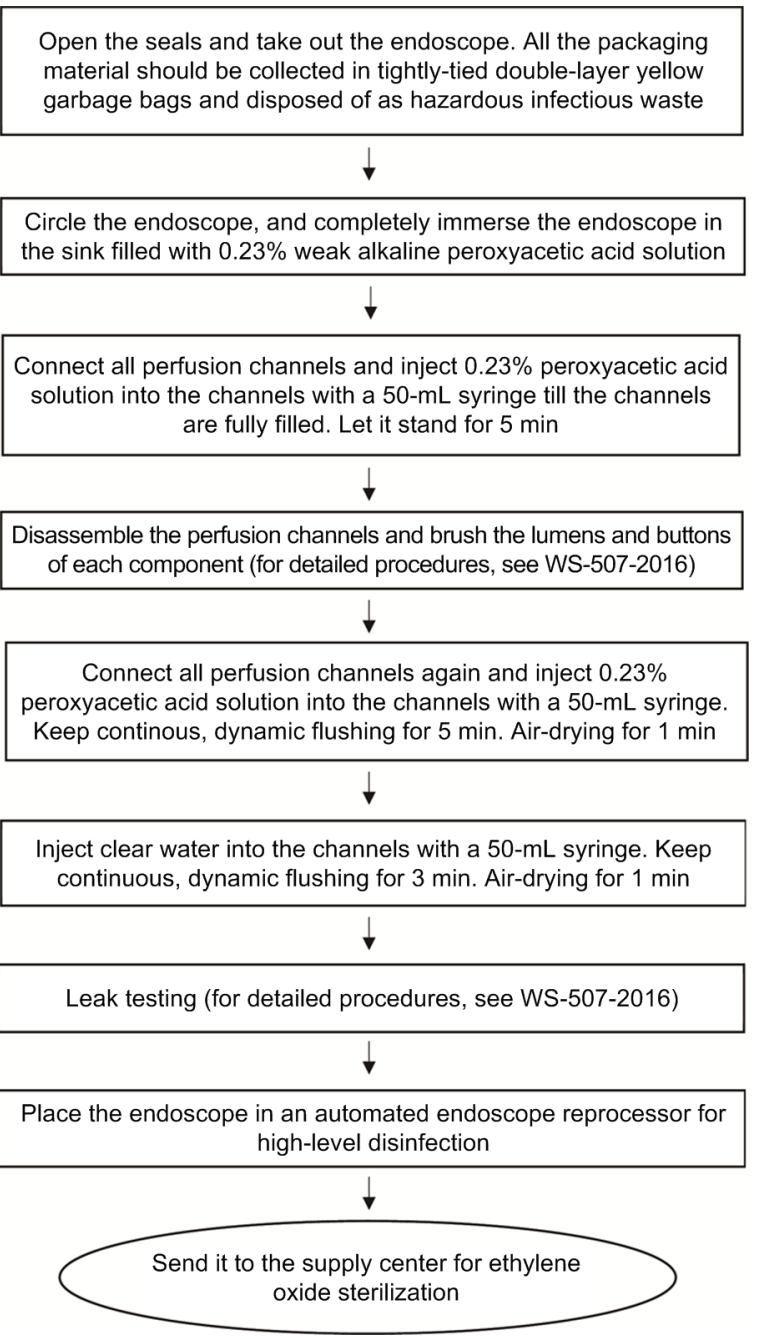

Fig. 2 Workflow of cleaning, disinfection, and sterilization of endoscopes contaminated by patients infected with COVID-19

\subsection{Improved strategies for medical waste disposal}

We have improved medical waste disposal workflow as follows: all the packaging materials and all the medical wastes generated in treatment or reprocessing should be placed in double-layer yellow plastic bags tied in a gooseneck shape with white sealing bands. Repeat the packaging procedures till the waste is wellwrapped in four layers of yellow plastic bags without puncture or damage. Apply $2500 \mathrm{mg} / \mathrm{L}$ available chlorine disinfectant to the opening and then place it into a leakproof transport container with clear, special infectious markers (white bands affixed, information required such as facility name, date, type of waste, ward location, etc.) (Fig. S3).

\section{Evaluation}

No occupational infection was reported in our hospital following the workflow stated above. Multiple samples collected from the surfaces or lumens of the endoscopes showed negative results in 2019 novel coronavirus (2019-nCoV) nucleic acid tests and no bacterial colonies.

\section{Discussion}

\subsection{Endoscopy is indispensable in the treatment of severe COVID-19 cases}

Severe COVID-19 cases have a higher risk of regurgitation and aspiration, and are susceptible to various complications such as ventilator-associated pneumonia, catheter-related blood infections, stress ulcers, and other common adverse events in the ICU. According to the interim guidance for clinical management of severe acute respiratory infection suspected with COVID-19 issued by WHO (2020), it is advisable to provide enteral nutrition as early as possible within 24 to $48 \mathrm{~h}$ after admission to reduce the incidence of stress ulcers and gastrointestinal bleeding. Therefore, it is of great clinical significance to place jejunal nutrition tubes in these patients to improve their overall nutritional status, and to perform emergency bedside endoscopic hemostasis when necessary to improve prognosis and reduce mortality. In our hospital, we placed jejunal nutrition tubes in the 12 severe COVID-19 cases and performed endoscopic hemostasis for them. As one of the cases was complicated by lower gastrointestinal bleeding, we relieved the intestinal obstruction and did hemostasis with titanium clips by bedside colonoscopy, which rendered the patient in a relatively stable condition for subsequent treatment. A preliminary assessment of these patients with nutrition tubes showed an improved nutritional status, which requires future follow-up assessment as the disease progresses.

\subsection{Importance of bedside pre-processing and special transport workflow in actual clinical work}

Despite the term "not recommending bedside preprocessing" included in the recommendations by CSDE (2020b), we found in actual clinical practice that there could be some problems without pre-processing. 
For instance, there could be a higher risk of environment contamination through unexpected physical contact, and organics, blood, and other contaminants could easily dry up in the endoscope lumens, leading to greater difficulty in cleaning and disinfection. Therefore, we have made some improvements, including the procedures of endoscope reprocessing and the selection of chemicals, to the workflow proposed by NHFPC (2017a) to better meet the needs in our actual clinical practice. In order to reduce the risk of cross-contamination during transport, taking into account multiple factors including the potential contamination of the endoscope-related equipment, we set up a special route to transfer the equipment from bedside to the endoscope reprocessing center.

\subsection{Peroxyacetic acid $(0.23 \%)$ can serve as high- level disinfectant for COVID-19}

The $0.23 \%$ peroxyacetic acid solution adopted by our hospital is weak alkaline activator solution diluted from sterilizing powder, instead of conventional peroxyacetic acid. It has been proved by multi-center clinical studies and by the national and provincial Centers for Disease Control and Prevention (CDCs) that it performs well in disinfecting pathogenic microorganisms, decomposing organics, and removing biomembrane without damage to the endoscopes. When the sterilizing powder dissolves in water, peroxyacetic acid is generated. This exerts a strong oxidizing and decomposing effect on organics and proteins. Meanwhile, alkaline sodium is produced, which acts as an alkaline cleaning agent (Gu et al., 2018). Lipid solvents such as chlorine-containing disinfectant and peracetic acid have been reported to effectively inactivate the 2019-nCoV (NHC, 2020a). The $0.23 \%$ peroxyacetic acid solution we utilized in clinical practice could meet the requirements of highlevel disinfection for the virus.

\subsection{Improved workflow worthy to be recommended}

The workflow we provide here, from personnel training, item preparation, endoscopic operations, bedside endoscope pre-processing, leakproof transport of contaminated endoscopes, to endoscope cleaning, disinfection, and sterilization in the reprocessing center, strictly abides by the rules of infection control in health care facilities. In accordance with the hy- gienic standard for disinfection in hospitals (AQSIQ, 2012), qualification assessments on the reprocessed endoscope in our center showed negative results in 2019-nCoV nucleic acid tests and no bacterial colonies. Hereby, we provide an effective, safe, operationfriendly, and adaptive working system for endoscopic reprocessing after bedside endoscopic operations in the context of the COVID-19 pandemic for reference.

\section{Conclusions}

Endoscopic diagnosis and treatment play an important role in improving the prognosis and reducing the mortality of severe COVID-19 cases. Amidst the COVID-19 pandemic, it is of utmost importance for health care facilities to ensure personnel protection and prevent cross-infection during bedside endoscopic operations. We summarized our nursing experience on endoscope reprocessing after bedside endoscopic diagnosis and treatment on severe COVID-19 patients. However, the additional disinfection steps might add to the deterioration of the endoscopes during reprocessing. Further evidencebased studies are anticipated to consummate the workflow in the future.

\section{Contributors}

Conceived and designed the study: Qing GU, Hua-fen WANG, and Zhe SHEN. Collected the data: Qing GU, Ying FANG, Zhe SHEN, Yan WANG, and Xin WU. Analyzed the data: Qing GU, Ying FANG, Ye LU, Zhe SHEN, Yan WANG, Xin WU, and Li CEN. Wrote the paper: Qing GU, Hua-fen WANG, Ying FANG, Ye LU, Zhe SHEN, Yan WANG, Xin WU, Li CEN, and Yi-shu CHEN. All authors have read and approved the final manuscript and, therefore, have full access to all the data in the study and take responsibility for the integrity and security of the data.

\section{Compliance with ethics guidelines}

Qing GU, Hua-fen WANG, Ying FANG, Ye LU, Zhe SHEN, Yan WANG, Xin WU, Li CEN, and Yi-shu CHEN declare that they have no conflict of interest.

All procedures followed were in accordance with the ethical standards of the responsible committee on human experimentation (institutional and national) and with the Helsinki Declaration of 1975, as revised in 2008 (5). Informed consent was obtained from all patients for being included in the study. Additional informed consent was obtained from all patients for whom identifying information is included in this article. 


\section{References}

AQSIQ (General Administration of Quality Supervision, Inspection and Quarantine of the People's Republic of China), 2012. Hygienic Standard for Disinfection in Hospitals, GB 15982-2012. National Standards of People's Republic of China (in Chinese).

Bakanidze L, Imnadze P, Perkins D, 2010. Biosafety and biosecurity as essential pillars of international health security and cross-cutting elements of biological nonproliferation. BMC Public Health, 10(Suppl 1):S12. https://doi.org/10.1186/1471-2458-10-S1-S12

CSDE (Chinese Society of Digestive Endoscopology), 2020a. Guiding principles on gastrointestinal endoscopic diagnosis and treatment during the prevention and control of COVID-19 by the Chinese Society of Digestive Endoscopology. http://www.csde.org.cn/news/detail.aspx? article id $=2883$ [Accessed on Apr. 3, 2020] (in Chinese).

CSDE, 2020b. Recommendations for endoscope cleaning, disinfection and sterilization in gastrointestinal endoscopy center during the prevention and control of COVID-19 by the Chinese Society of Digestive Endoscopology. http://www.csde.org.cn/news/detail.aspx?article_id=2884 [Accessed on Apr. 3, 2020] (in Chinese).

Emergency Care Research Institute, 2018. Top 10 Health Technology Hazards for 2019. https://www.ecri.org/ Resources/Whitepapers_and_reports/Haz_19.pdf [Accessed on Apr. 3, 2020].

Fang Y, 2011. Practical Endoscopic Cleaning and Disinfection Techniques. Zhejiang University Press, Hangzhou, China (in Chinese).

Gu Q, Xu M, Sheng TT, et al., 2018. The cleaning effect of peroxyacetic acid sterilizing powder on organics on endoscopes. Nurs Rehabil J, 17(2):69-70 (in Chinese).

Gu Q, Lin YM, Cen L, et al., 2019. Endoscopic ultrasonography is useful in the diagnosis and treatment of rectal neuroendocrine neoplasms: a case series. $J$ Zhejiang Univ-Sci B (Biomed \& Biotechnol), 20(10):861-864. https://doi.org/10.1631/jzus.B1900168

Huang CL, Wang YM, Li XW, et al., 2020. Clinical features of patients infected with 2019 novel coronavirus in Wuhan, China. Lancet, 395(10223):497-506. https://doi.org/10.1016/S0140-6736(20)30183-5

Lou SM, Zhang M, Wu ZR, et al., 2019. Combined gastroscopic and choledochoscopic transabdominal nasobiliary drainage. J Zhejiang Univ-Sci B (Biomed \& Biotechnol), 20(11):940-944. https://doi.org/10.1631/jzus.B1900060

NHC (National Health Commission of the People's Republic of China), 2020a. Guideline for diagnosis and treatment of COVID-19 (the sixth trial version). http://www.nhc. gov.cn/yzygj/s7653p/202002/8334a8326dd94d329df351d 7da8aefc2.shtml [Accessed on Apr. 3, 2020] (in Chinese).

NHC, 2020b. Guidelines for the prevention and control of COVID-19 in health care facilities (the first version). http://www.nhc.gov.cn/yzygj/s7659/202001/b91fdab7c3 04431 eb082d67847d27e14.shtml [Accessed on Apr. 3, 2020] (in Chinese).

NHFPC (National Health and Family Planning Commission of the People's Republic of China), 2017a. Regulation for
Cleaning and Disinfection Technique of Flexible Endoscope, WS 507-2016. National Standards of People's Republic of China (in Chinese).

NHFPC, 2017b. Regulation for Prevention and Control of Healthcare Associated Infection of Airborne Transmission Disease in Healthcare Facilities, WS/T 511-2016. National Standards of People's Republic of China (in Chinese).

WHO (World Health Organization), 2020. Clinical management of severe acute respiratory infection when COVID19 is suspected. Interim guidance. https://www.who.int/ publications-detail/clinical-management-of-severe-acuterespiratory-infection-when-novel-coronavirus-(ncov)infection-is-suspected [Accessed on Apr. 3, 2020].

Wu JT, Leung K, Leung GM, 2020. Nowcasting and forecasting the potential domestic and international spread of the 2019-nCoV outbreak originating in Wuhan, China: a modelling study. Lancet, 395(10225):689-697. https://doi.org/10.1016/S0140-6736(20)30260-9

Xia TT, Shi S, Yang JY, et al., 2019. The latest developments of flexible endoscopes reprocessing in China and other countries. Chin J Nosocomiol, 29(8):1272-1277 (in Chinese).

\section{List of electronic supplementary materials}

Fig. S1 Treatment of contaminated endoscopy during beside pre-processing

Fig. S2 Treatment of perfusion channels

Fig. S3 Transport of contaminated endoscopes

\section{中文概要}

题 目: 危重型新型冠状病毒肺炎患者床边内镜诊疗后内 镜再处理流程改良分析

目 的: 在疫情期间, 需要通过保证内镜处理质量, 避免 过程环节操作中的接触污染, 防止气溶胶的散 发, 从而保护从业人员, 杜绝院内感染。

创新点: 内镜诊疗对改善重症患者预后及降低患者死亡率 有重要作用。该研究依据实际临床工作特质, 对 内镜再处理过程中的部分方法及化学清洗消毒 剂的选择进行了改良与替换。

方 法: 从 12 例重症患者行 23 次内镜诊治后的内镜处理 操作中提取经验, 结合相关指南规范, 提出了新 型冠状病毒肺炎患者床边内镜诊疗后内镜再处 理改良流程。

结 论: 内镜诊疗在重症及危重症患者治疗中不可或缺。 临床工作中床旁预处理操作及专线转运流程对 于预防院内感染具有重要意义。替换后的化学清 洗消毒剂（0.23\%过氧乙酸）对新冠病毒可达到 高水平消毒。改良后的床边内镜诊疗后内镜再处 理操作流程顺畅, 感染控制严谨, 值得临床进一 步推广。

关键词: 新型冠状病毒 (COVID-19)；2019-nCoV; 消毒; 消化内镜; 防护流程 